\title{
Palisaded Lymph Node Myofibroblastoma
}

National Cancer Institute

\section{Source}

National Cancer Institute. Palisaded Lymph Node Myofibroblastoma. NCI Thesaurus. Code C6584.

A myofibroblastoma arising from the inguinal lymph nodes. It is characterized by the presence of nuclear palisading. 